\title{
Guillain-Barre Syndrome as a Paraneoplastic Syndrome from Ewing Sarcoma
}

\author{
Oakes MH', Davis $\mathrm{LE}^{2}$ and Ryan CW'* \\ ${ }^{1}$ Department of Medicine, Oregon Health \& Science \\ University, United States \\ ${ }^{2}$ Oregon Health \& Science University, Knight Cancer \\ Institute, United States \\ *Corresponding author: Ryan CW, Knight Cancer \\ Institute, Oregon Health \& Science University, 3485 S \\ Bond Ave Building 2, Portland, OR 97239, United States
}

Received: March 12, 2021; Accepted: March 30, 2021;

Published: April 06, 2021

\begin{abstract}
Background: Paraneoplastic syndromes have been associated with certain malignancies, classically with small cell lung cancer. However, there have been case reports of solid tumors that have been associated with paraneoplastic syndromes as well. This is believed to be the first case that details a young man with Ewing sarcoma who developed Guillain-Barre Syndrome in the form of a paraneoplastic syndrome.
\end{abstract}

Case Presentation: A 23-year-old man with localized Ewing sarcoma presented to the hospital with weakness. He was initially treated with interval compressed chemotherapy and radiation, but developed recurrence and underwent hemipelvectomy. He had chronic numbness of his extremities from vincristine that felt acutely worsened over several days prior to admission. Additionally, he was now having difficulty grasping objects and walking. His exam was notable for mildly decreased strength in his lower extremities but preserved in his upper extremities along with intact extraocular movements. The following morning he was unable to move and lost all sensation in his left lower extremity along with new dysphagia. Urgent work-up revealed a normal MRI brain, MRI spine with enhancement of cauda equina nerve roots around the conus, and a lumbar puncture with cytoalbuminologic dissociation. Ultimately was diagnosed with presumed Guillain-Barre Syndrome and treated with IVIG.

Conclusion: Guillain-Barre is a rare but serious syndrome that needs to be recognized quickly given its high mortality and morbidity rate and necessity of quick intervention. It is imperative for clinicians to maintain a high degree of clinical suspicion as a manifestation of paraneoplastic syndrome from malignancies.

Keywords: Guillain-barre syndrome; paraneoplastic syndrome; Ewing sarcoma; Acute inflammatory demyelinating syndrome

\section{Background}

Paraneoplastic syndromes can be classified according to the organ system they involve, such as endocrine, neurologic, hematologic, and dermatologic syndromes. Neurologic paraneoplastic syndromes have a number of manifestations and are associated with different primary malignancies, such as limbic encephalitis (small cell lung cancer), cerebellar degeneration (small cell lung cancer), myasthesia gravis (thymoma), and autonomic neuropathy (small cell lung cancer and thymoma). Various onconeural antibodies have been associated with these syndromes, with anti-amphiphysin, anti-CV2, anti-Hu, anti-Ma2, anti-recoverin, anti-Ri, and anti-Yo being the most wellknown [1]. Guillain-Barre Syndrome has been reported as a rare paraneoplastic syndrome, primarily in the setting of lung cancer and lymphoma. Notably, in several of these cases, antibody testing was negative [2-5]. It is unclear why certain malignancies have a greater predisposition to paraneoplastic syndromes. One hypothesis involves the cellular origin with cancers that express neuroendocrine proteins or originate from immunoglobulin-producing cells as being more likely to be associated [6]. In contrast, Ewing sarcoma is not commonly associated with paraneoplastic syndromes. Its histogenesis has been widely debated with some hypothesizing that it originates from mesenchymal stem cells, which would possibly explain why it is less likely associated with these syndromes. On the other hand, there are those that believe its lineage arises from neural crest cells, which one would expect would make it more predisposed to neurologic paraneoplastic syndromes [7]. The few cases of paraneoplastic syndrome with Ewing sarcomas that have been reported have been endocrinopathies involving Cushing's syndrome and ectopic ACTh release [8-10]. This case is believed to be the first to have an association between Ewing sarcoma and Guillain-Barre Syndrome.

\section{Case Presentation}

A 23-year-old man with localized Ewing sarcoma of the left pelvic ramus presented to the hospital with weakness. He was initially treated with interval compressed VAC/IE (vincristine, doxorubicin, cyclophosphamide, ifosfamide, etoposide) and radiation therapy, but subsequently developed recurrence a year later and underwent hemipelvectomy. He had chronic numbness of his bilateral hands and extremities attributed to his previous chemotherapy, but felt that these symptoms had acutely worsened over several days prior to admission. In addition, he was now having a hard time grasping objects and difficulty walking and nearly had a ground level fall. Additional review of systems revealed significant fatigue, decreased bowel movements, and photophobia with eye pain but no vision
Sarcoma Res Int - Volume 6 Issue 1 - 2021

Submit your Manuscript | www.austinpublishing group.com

Ryan et al. @ All rights are reserved
Citation: Oakes MH, Davis LE and Ryan CW. Guillain-Barre Syndrome as a Paraneoplastic Syndrome from Ewing Sarcoma. Sarcoma Res Int. 2021; 6(1): 1046. 
changes.

His initial vitals were temperature 37.6 degrees Celsius, heart rate 66 beats per minute, blood pressure $110 / 65 \mathrm{~mm} \mathrm{Hg}$, and respiratory rate 13 breaths per minute. Physical exam was notable for an uncomfortable appearing man with $4+/ 5$ strength in his left lower extremity and $4 / 5$ strength in his right lower extremity, 5/5 strength in bilateral upper extremities, and intact extraocular movements. His initial chemistry and complete blood counts were unremarkable. The initial impression of his presentation was attributed to malnutrition and poor oral intake in setting of chronic known neuropathy from his chemotherapy.

The next morning he was unable to move and lost all sensation in his left lower extremity along with new dysphagia. On exam, he had $0 / 5$ strength in his left lower extremity, $2 / 5$ strength in his right lower extremity, and reflexes were unable to be elicited in his lower extremities. He underwent urgent MRI of his brain and total spine. MRI brain revealing no acute abnormalities or metastatic disease; MRI spine showed smooth increased enhancement of cauda equina nerve roots around the conus indicating a possible inflammatory or demyelinating etiology. A lumbar puncture was then obtained revealing a cytoalbuminologic dissociation with cytology negative for malignancy and cultures with no growth. He was subsequently started on empiric dexamethasone, but by nighttime, he developed increasing respiratory distress ultimately requiring intubation. Given his rapidly ascending sensorimotor deficits and findings on lumbar puncture, he was diagnosed with presumptive Guillain-Barre Syndrome with acute inflammatory demyelinating syndrome subtype and subsequently started on a five-day course of IVIG. EMG/NCS was performed several days later confirming Guillain-Barre Syndrome. Antibody testing with neurofascin 140, neurofascin 155, contactin-1, and anti-ganglioside later all resulted negative.

The underlying etiology of this patient's Guillain-Barre Syndrome was ultimately suspected to be the result of a paraneoplastic syndrome from his recurrent Ewing Sarcoma. There was no preceding gastrointestinal illness or travel history to believe that this was attributable to Campylobacter. Viral infections have been associated with Guillain-Barre as well, but extensive collateral history obtained from family revealed that the patient had no antecedent upper respiratory symptoms and had negative hepatitis serologies [11]. The patient was started on a regimen of cyclophosphamide and topotecan to treat the recurrent Ewing sarcoma as there were no other identifiable causes for his Guillain-Barre.

\section{Discussion and Conclusions}

This is believed to be the first case of Guillain-Barre Syndrome associated with Ewing sarcoma and emphasizes the importance of recognizing the clinical syndrome given its poor prognosis with mortality rates ranging from 3-7 \% and $20 \%$ of patients being unable to walk after six months [12]. While 30-40\% of Guillain-Barre Syndrome cases are classically associated with Campylobacter jejuni, only 0.1-1 \% of Campylobacter infections actually result in GuillainBarre Syndrome [13]. At the time of this manuscript, the patient was discharged to a long-term acute care facility and was tolerating tracheostomy collar trials. It is difficult to determine whether this improvement is from the chemotherapy for his Ewing sarcoma or the IVIG, which is standard of care for Guillain-Barre Syndrome.

\section{References}

1. Pelosof LC, Gerber DE. Paraneoplastic syndromes: an approach to diagnosis and treatment. Mayo Clin Proc. 2010; 85: 838-854.

2. Kim MH, Hwang MS, Park YK, Park Y, Ahn YC, Oh HS, et al. Paraneoplastic Guillain-Barré Syndrome in Small Cell Lung Cancer. Case Rep Oncol. 2015; 8: 295-300.

3. Wu D, Liu A, Baldinger E, Frontera AT. A Case of Paraneoplastic GuillainBarré Syndrome Associated with Squamous Cell Carcinoma of the Lung. Cureus. 2018; 10: e3202.

4. Deb B, Pandey MR, Torka P, Sundaram S. Diffuse large B-Cell lymphoma associated with paraneoplastic Guillain-Barré syndrome: A diagnostic and therapeutic challenge. Hematol Oncol Stem Cell Ther. 2020: S16583876(20)30106-0.

5. Patil M, Muppidi V, Meegada S, Dowell KT, Bowers JD. Guillain-Barre Syndrome and Syndrome of Inappropriate Antidiuretic Hormone (SIADH) Secretion as Paraneoplastic Syndromes in Splenic Marginal B-cell NonHodgkins Lymphoma: A Rare Presentation. Cureus. 2020; 12: e10133.

6. Dalmau J, Rosenfeld MR. Paraneoplastic syndromes of the CNS. Lancet Neurol. 2008; 7: 327-340.

7. Tu J, Huo Z, Gingold J, Zhao R, Shen J, Lee DF. The Histogenesis of Ewing Sarcoma. Cancer Rep Rev. 2017; 1: 10.15761/CRR.1000111.

8. Di Ruscio V, Del Baldo G, De Pasquale MD, De Vito R, Miele E, Colafati GS, et al. Ectopic ACTH Secretion in a Child With Metastatic Ewing Sarcoma: A Case Report. Front Oncol. 2020; 10: 574.

9. Preeyasombat C, Sirikulchayanonta $V$, Mahachokelertwattana $P$, Sriphrapradang A, Boonpucknavig S. Cushing's syndrome caused by Ewing sarcoma secreting corticotropin releasing factor-like peptide. Am J Dis Child. 1992; 146: 1103-1105.

10. Guran T, Turan S, Ozkan B, Berrak SG, Canpolat C, Dagli T, Eren FS, Bereket A. Cushing's syndrome due to a non-adrenal ectopic adrenocorticotropinsecreting Ewing sarcoma in a child. J Pediatr Endocrinol Metab. 2009; 22: 363-368.

11. Malek E, Salameh J. Guillain-Barre Syndrome. Semin Neurol. 2019; 39: 589595.

12. Willison $\mathrm{HJ}$, Jacobs $\mathrm{BC}$, van Doorn PA. Guillain-Barré syndrome. Lancet. 2016; 388: 717-727.

13. Jasti AK, Selmi C, Sarmiento-Monroy JC, Vega DA, Anaya JM, Gershwin ME. Guillain-Barre syndrome: causes, immunopathogenic mechanisms and treatment. Expert Rev Clin Immunol. 2016; 12: 1175-1189. 